\title{
CT-Scan Fat Halo Sign in a Patient with Abdominal Mass and Constipation
}

\author{
Giovanni Marasco, Rocco Maurizio Zagari, Franco Bazzoli
}

Department of Medical and Surgical Sciences, University of Bologna, Bologna, Italy

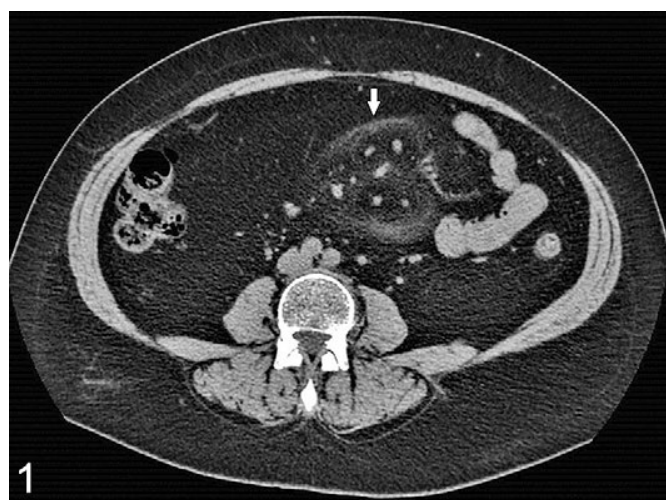

A 59-year-old woman referred to our Gastroenterology Unit for constipation and bloating since one year, without history of weight or appetite loss. She had no comorbidities and a history of hystero-annessectomy for uterine fibromatosis. Laboratory examinations (i.e. leucocyte count, $\mathrm{C}$ reactive protein, oncomarkers) were normal. At abdominal ultrasound (US), a hypoechoic mesenterial mass lipoma-like was highlighted. The computed tomography (CT)-scan with contrast showed a blurred increase in density and a thickening of the mesenteric adipose tissue with centimetric multiples lymph nodes (the largest with a diameter of $34 \mathrm{~mm}$ ) circumscribed by dense tissue bands, the typical "fat halo sign"(Fig. 1, arrow). This image, also known as the "fat halo sign", describes the preservation of the fat surrounding mesenteric vessels and lymph nodes due to a diffuse fat stranding in patients with sclerosing mesenteritis (SM). An US-guided biopsy specimen from the thickened massforming mesenteric adipose tissue was obtained. The diagnosis of SM was confirmed by histology: chronic inflammation and fat necrosis depicted by Masson's Trichrome staining (Fig. $2,10 x)$ with numerous lipid laden macrophages, stained by immunohistochemistry with CD68 antibodies (Fig. 3, 10x). The patient was treated with oral prednisone $(0.5 \mathrm{mg} / \mathrm{kg} /$ day $)$ for 6 months and, thereafter, with oral budesonide for a further 18 months, achieving resolution of symptoms.

Sclerosing mesenteritis is an idiopathic inflammatory disease affecting the mesentery of the small bowel. Most patients are asymptomatic, while some patients refer a wide spectrum of symptoms such as abdominal pain, bloating, altered bowel habits, vomiting and fever [1]. The CT sign helps distinguishing this pathological entity from more aggressive entities such as lymphoma, carcinoid tumour or carcinomatosis [2,3]. A recent study [4] concluded that the pathognomonic CT features of SM were the fat halo sign and the presence of a hyperdense capsule; additional features were the presence of a mass lesion mainly composed by fat and having inhomogeneous structure.

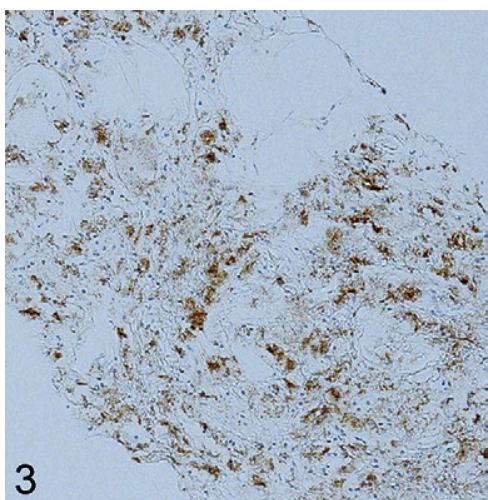

However, in most case series the diagnosis was confirmed by biopsy [1], similar to our experience. Since the clinical pattern and the diagnostic features of this rare condition have not been fully elucidated and could be similar to those of neoplastic conditions, biopsy is recommended [5].

The treatment of SM depends on several factors. As this is a self-limiting condition, in case of asymptomatic patients and a well-established diagnosis, a follow-up seems to be the best choice [1]. The medical treatment should be reserved for symptomatic non-surgical patients. The benefit of steroids and, less frequently, of colchicine, tamoxifen, 6-mercaptopurine, antibiotics, azathioprine, methotrexate or infliximab was reported [5]. Surgical intervention should be reserved for cases of non-treatable bowel or vessels obstruction.

Corresponding author: Giovanni Marasco, giovannimarasco89@gmail.com

Conflicts of interest: None to declare.

\section{REFERENCES}

1. Akram S, Pardi DS, Schaffner JA, Smyrk TC. Sclerosing mesenteritis: clinical features, treatment, and outcome in ninety-two patients. Clin Gastroenterol Hepatol 2007;5:589-596. doi:10.1016/j.cgh.2007.02.032

2. Horton KM, Lawler LP, Fishman EK. CT findings in sclerosing mesenteritis (panniculitis): spectrum of disease. Radiographics 2003;23:1561-1567. doi:10.1148/rg.1103035010

3. Lucey BC, Stuhlfaut JW, Soto JA. Mesenteric lymph nodes seen at imaging: causes and significance. Radiographics 2005;25:351-365. doi:10.1148/rg.252045108

4. Coulier B. Mesenteric panniculitis. Part 2: prevalence and natural course: MDCT prospective study. JBR-BTR 2011;94:241-246.

5. Sharma P, Yadav S, Needham CM, Feuerstadt P. Sclerosing mesenteritis: a systematic review of 192 cases. Clin J Gastroenterol 2017;10:103-111. doi:10.1007/s12328-017-0716-5 